\title{
Tracking the Effects of Rigidity Percolation Down to the Liquid State: Relaxational Dynamics of Binary Chalcogen Melts
}

\author{
F. J. Bermejo and C. Cabrillo \\ Instituto de Estructura de la Materia, CSIC, and Department Electricidad y Electrónica-Unidad Asociada CSIC, \\ Facultad de Ciencia y Tecnología, Universidad del País Vasco/EHU, P. Box 644, E-48080-Bilbao, Spain \\ E. Bychkov \\ Université du Littoral, 59140 Dunkerque, France \\ P. Fouquet, G. Ehlers, ${ }^{*}$ and W. Häussler ${ }^{\dagger}$ \\ Institut Laue Langevin, BP 156, 38042 Grenoble Cedex 9, France \\ D. L. Price and M. L. Saboungi \\ Centre de Recherche sur les Matériaux à Hautes Températures and Centre de Recherche sur la Matière Divisée, \\ 45071 Orléans Cedex 2, France \\ (Received 29 January 2008; published 20 June 2008)
}

\begin{abstract}
The stochastic dynamics of binary liquids with formula $A_{x} B_{1-x}, x=0-0.4$ is investigated by neutron spin-echo spectroscopy. These compositions comprise samples of varying chemical connectivity, ranging from twofold-coordinated liquid Se to higher average coordinated $\mathrm{As}_{2} \mathrm{~S}_{3}$. The parameters giving the temperature dependence of the relaxation patterns show a quasilinear dependence on the average coordination number. The results thus extend the validity of the rigidity concept into the normal liquid state and emphasize the role played by the fine details of atomic bonding on the dynamics at $10 \mathrm{ps}-1 \mathrm{~ns}$ scales.
\end{abstract}

DOI: 10.1103/PhysRevLett.100.245902

The concept of rigidity percolation was coined some 25 years ago [1] to explain how an elastic network goes from a mobile state into a rigid domain as constraints are added to it. The development of this model was motivated by the need to understand the elastic behavior of covalent glasses such as the chalcogenide family [2]. In this class of materials, covalent central bond stretching and Keatingtype bond-angle bending forces are much stronger than those due to van der Waals or electrostatic interactions. A mean coordination number is defined as $\langle r\rangle=n_{A} x+$ $2(1-x)$ for systems of general formula $A_{x} B_{1-x}$, where A is a species with coordination number $n_{A}=4$ or 3 (such as Ge or As) and $B$ is the twofold-coordinated counterpart (Se or S). The rigidity of the glassy network is known to be dependent on $\langle r\rangle$, and the number of constraints $N_{c}$ acting on the atoms composing the glassy matrix is given by $N_{c}=$ $\sum_{r=2}^{4} n_{r}[1 / 2+(2 r-3)]$, where $n_{r}$ stands for the number of atoms with coordination $r=2,3,4$ and the last term corresponds to the angular constraints [2].

Recent theoretical advances have been registered [3-9], in part as a result from cross fertilization from developments within subdisciplines such as the physics of granular matter $[3,6]$, and also from careful studies on simple model systems such as those composed by particles interacting via square-well potentials [8]. Particular emphasis has been put on studies on the microscopic mechanisms causing rigidity and, in fact, a good number of questions on the way force propagates in amorphous matter and how force propagation evolves towards the elastic continuum when the coordination number increases, still remain open [3].
PACS numbers: 66.10. $-\mathrm{x}, 65.20 . \mathrm{Jk}, 66.25 .+\mathrm{g}, 66.70 .-\mathrm{f}$

Some approaches establish a connection between the glassforming tendency and the connectivity of the network $[9,10]$, which is grounded on the fact that flexible systems exhibit, in their glass phase, a number of low-frequency excitations called floppy modes that provide channels in the energy landscape, such that entropy and fragility of the glass depend on the connectivity of the supercooled melt. The fraction of floppy modes $f$ is given by $f=2-5\langle r\rangle / 6$ and a rigidity transition takes place for $f=0$, setting the critical value $\left\langle r_{c}\right\rangle=2.4$. Following a quite different spirit, recent developments, usually referred to as elastic models [5], are conceptually related to the cherished free-volume theories in the sense that they assume that the activation energy for liquid flow motions is determined by a physical quantity which, in contrast with the configurational entropy, can be measured on short time scales. Furthermore, recent reports [11] have evidenced striking correlations between some elastic properties such as the Poisson ratio and the atomic packing density or the glass-network dimensionality as measured by the average coordination.

Experiments concerning the thermodynamic properties of the Se-As-Ge ternary system [12] such as the excess heat capacity and the activation energy for viscous flow have shown a clear correlation of these quantities with the bond connectivity, which evidences that the effects of rigidity can also be investigated within the liquid state. Here, we report experimental results that go one step further and clearly show a quasilinear dependence of microscopic parameters characterizing the melt dynamics on the average coordination number. 
The microscopic dynamics were studied using neutron spin-echo (NSE) spectroscopy, which gives the decay of the intermediate scattering function $I(Q, t)$ as a function of momentum transfer $Q$ and time $t$. From such studies, relevant parameters such as the activation energy for the main structural relaxation time as well as a coefficient measuring the temperature dependence of the deviation from exponential relaxation, i.e., the stretching parameter $\beta$ in the Kohlrausch-Williams-Watts (KWW) function, were derived and their dependence on the average liquid connectivity as quantified by $\langle r\rangle$, explored in detail. The experiments here reported on were carried out on binary $A_{x} B_{1-x}$ glasses with average coordination $\langle r\rangle$ in the range 2.0-2.4. The samples were prepared by rapid quenching from the melts. The set of compositions for which clear echos could be observed within our instrumental configuration were $\mathrm{As}_{x} \mathrm{Se}_{100-x}$ with $x=0,12$, and 25, $\mathrm{Se}_{x} \mathrm{Ge}_{100-x}$ with $x=100,80$, and 92, and $\mathrm{As}_{2} \mathrm{~S}_{3}$. Some samples with different compositions and larger values of $\langle r\rangle$ were also explored, but did not provide data over a wide enough range of temperature.

The experiments were carried out using the neutron spin-echo spectrometer IN11 at the Institut Laue Langevin, Grenoble, using the $30^{\circ}$ detector option IN11C. The instrument was set up using an incident wavelength $\lambda=5.54 \AA$ and scattering angles between $60^{\circ}$ and $118^{\circ}$. This allowed us to explore a region of momentum transfers within $1.13 \leq Q \leq 1.94 \AA^{-1}$. This range spans the region of the first diffraction peak in the average static structure factor $S(Q)$, as measured by neutron or x-ray diffraction [13].

Because of the limitations in the available spectrometer time window ( $8 \mathrm{ps}-1.4 \mathrm{~ns}$ ) our measurements are necessarily limited to temperatures well above the liquid $\rightarrow$ glass transitions $T_{g}$. The temperature range where relaxation signals could be observed was approximately $1.2 \leq T_{g} \leq 1.9$.

Figure 1 displays selected NSE spectra which reveal a strong departure from the single-relaxation process expected for normal liquids. Such behavior is usually explained either by the presence of more than one relevant time scale or by the emergence of complex phenomena like those exhibited by glasses, where the relaxation times are continuously distributed according to a prescribed distribution function, yielding the well-known stretchedexponential law $\phi(t) \propto \exp \left[(-t \tau)^{\beta}\right]$. The appearance of stretched-exponential or KWW relaxation has also been ascribed to processes arising from a wide variety of physical origins [14,15].

Here, we have chosen a way of fitting the spectra in terms of relaxation patterns as simple as possible consistent with parameters that have a definite physical meaning. Accordingly, all spectra were analyzed with the stretchedexponential function, $S(Q, t)=A \exp \left[(-t / \tau)^{\beta}\right]$, where $A$, $\tau$, and $\beta$ were treated as adjustable parameters that yield a statistically sound representation of the relaxation patterns involved.

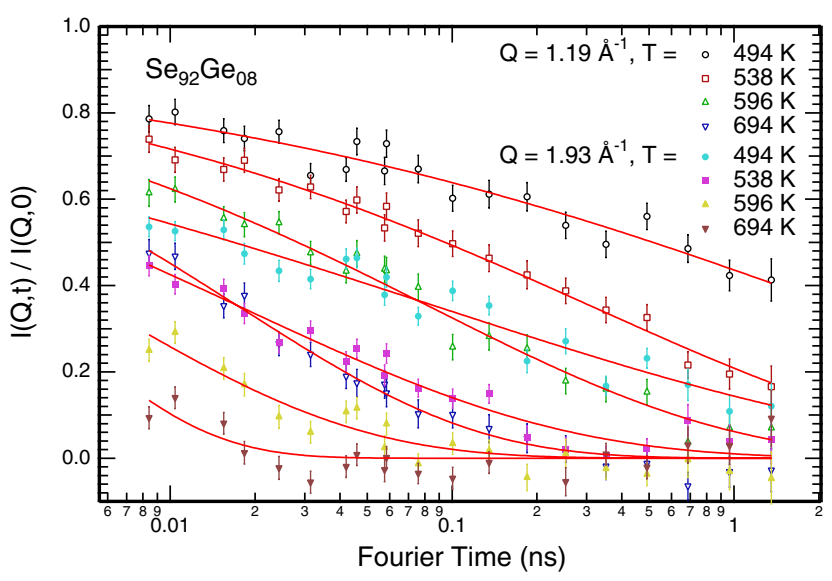

FIG. 1 (color online). Selected NSE spectra which were measured at the lowest and highest momentum transfer value, respectively. The data were measured on the $\mathrm{Se}_{92} \mathrm{Ge}_{08}$ sample. The solid lines are fits of KWW relaxation functions.

A sample of the obtained results for the temperature and wave-vector dependence of the relaxation times is given in Fig. 2. Our data display a strong dependence on wave vector which, contrary to results for other coherentscattering liquids $[16,17]$ which show a strong reduction of the spectral linewidth at wave vectors where $S(Q)$ shows its maxima, show only a weak hint of a relaxation time peak at such wave vectors [13]. The data were found to be well described by a simple Arrhenius temperature dependence given by $\tau(Q, T)=\tau_{0}(Q) \exp \left(E_{a} / k_{B} T\right)$, where $\tau_{0}$ and $E_{a}$ are the prefactor and activation energy, respectively, associated with liquid flow motions and $k_{B}$ is the Boltzmann constant. The simple Arrhenius description was chosen here as the measurements were taken at temperatures far away from the Kauzmann temperature, where relaxation times should finally diverge. We also fitted the data with Adam-Gibbs and VFT type equations, but the quality of the fit was only slightly improved ( $\chi^{2}$ reduced by less than $15 \%$ ) given the activation energies within error bars for reasonable assumptions of the Kauzmann temperature.

The data for $\mathrm{As}_{25} \mathrm{Se}_{75}$ show a much steeper temperature dependence than those for $\mathrm{Se}$ and $\mathrm{Se}_{92} \mathrm{Ge}_{08}$ : the latter show relaxation times that vary by about 4 orders of magnitude within some $200 \mathrm{~K}$ while the former display a variation of about 6 orders of magnitude within a range of about $320 \mathrm{~K}$. This result provides direct evidence of the remarkable dependence of thermal activation characteristics for particle diffusion on the average connectivity in the liquid, since the Se-Ge samples have an average coordination number $\langle r\rangle=2$ and 2.15 , respectively, compared with $\langle r\rangle=2.25$ for the As-Se samples.

Statistically sound estimates for $\tau_{0}$ were not obtained due to the scatter in the fitted data. The activation energies $E_{a}$, however, are less affected by statistical errors and are shown in Fig. 3. The plot shows a quasilinear dependence of the activation energy on $\langle r\rangle$. Interestingly, the data for $E_{a}$ 


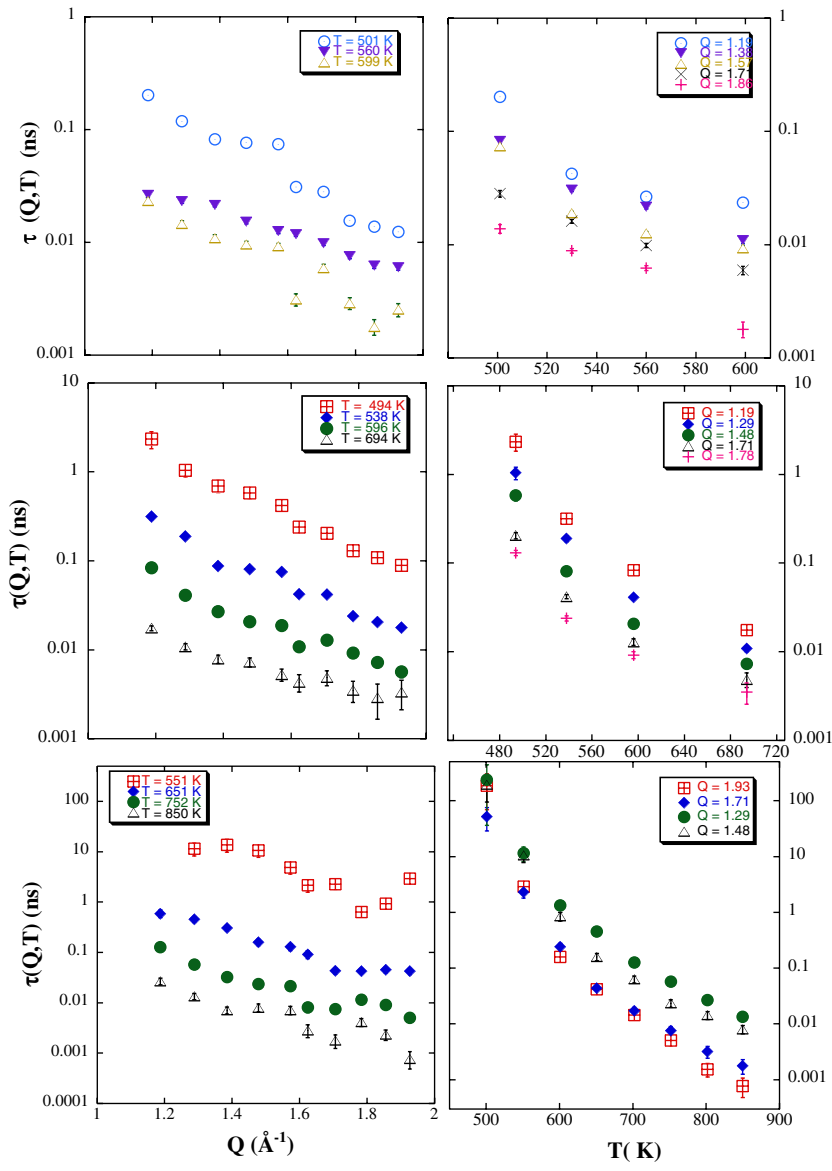

FIG. 2 (color online). Wave-vector dependence of the relaxation times for a set of temperatures (left-hand side graphs) and temperature dependence of the relaxation times for a set of wave vectors (right-hand side graphs). Data for liquid Se correspond to the two uppermost graphs. The wave-vector values are given in the insets in reciprocal angstrom units. Data for liquid $\mathrm{Se}_{92} \mathrm{Ge}_{08}$ are shown in the two middle frames. Data for liquid $\mathrm{As}_{25} \mathrm{Se}_{75}$ are given in the two lowest frames.

show a dependence on the average coordination number that is rather similar to the coordination number dependence of the glass-transition temperatures, as shown in the inset to Fig. 3.

The temperature dependence of the stretching parameter $\beta$ was also analyzed. Statistically significant results were found in the high-temperature region, where $\beta$ increased up to a value of 1 at high temperatures. An example of the quality of the derived data can be gauged from inspection of the inset of Fig. 4. There it is seen that both the values of the stretching parameter as well as their temperature dependences are significantly composition dependent and thus the value of the $\beta$ parameter is linked to the network connectivity. The temperature dependence was found to follow an exponential law

$$
\langle\beta\rangle_{Q}=\exp \left(-C_{\beta} / T\right)
$$

where the angular brackets stand for an average over the explored wave-vector range. The parameter $C_{\beta}$, character-

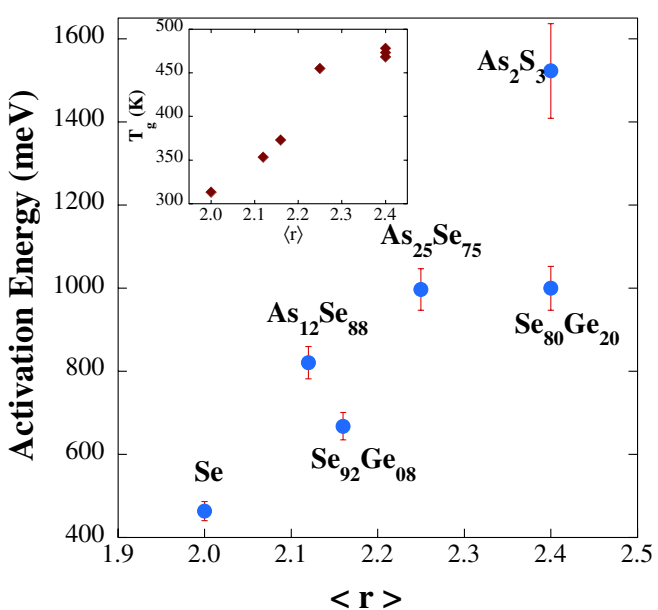

(Av. Coordination)

FIG. 3 (color online). The activation energies calculated from the Arrhenius plots versus average coordination number. The inset displays the dependence of the thermodynamic glasstransition temperature on the same parameter. Data pertaining the calorimetric glass-transition temperatures are taken from Ref. [2].

izing the strength of the temperature dependence of $\beta$, is shown in Fig. 4, again displaying a quasilinear dependence on $\langle r\rangle$.

The results shown in Fig. 4 show that, following some predictions [14], the deviation from exponential relaxation $(\beta=1)$ depends on the connectivity of the liquid. Moreover, $\beta$ decreases with increasing $\langle r\rangle$ which is understood within the "elastic model" $[18,19]$ in terms of an increased range of elastic interactions between local relaxation events. Such a range is defined in terms of a length $L=c_{s} \tau$, where $c_{s}$ stands for the sound velocity. The latter expression semiquantitatively accounts for the current findings, as can be inferred from data displayed in Fig. 2 which shows that $\tau$ increases with $\langle r\rangle$ and also from Fig. 3 which shows that the activation energy also increases with $\langle r\rangle$.

Our results thus extend into the stable liquid realm the validity of the relation between the low-frequency dynamics of covalent glasses - and thus the excess in configurational entropy - to the network connectivity as measured by the average coordination number $[7,20]$. They lend support to recent phenomenological treatments [20] that predict an explicit dependence of the energy term entering the VFT equation on the quantity $(7-5\langle r\rangle / 2)^{-1}$, making it to increase almost linearly as the coordination number increases. The current data also show common trends with those found for glasses using microscopic probes such as neutron [21] or Mössbauer [22] spectroscopies, where the inverse of the first and second spectral moments, which basically provide a measure of the dominance of low-frequency or floppy modes, show a smooth decrease with increasing coordination up to $\langle r\rangle=2.4$, where an illdefined threshold is reported. 


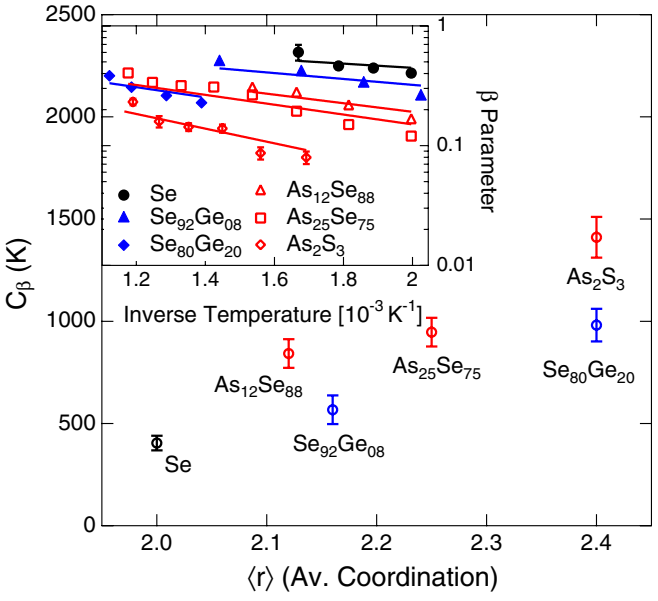

FIG. 4 (color online). Compositional dependence of the parameter $C_{\beta}$ characterizing the strength of the temperature dependence of $\beta$. The inset displays the temperature dependence of data pertaining to several different compositions.

The quasilinear dependence of the activation energy $E_{a}$ upon $\langle r\rangle$ is to be compared with activation energy data for the viscosity [12] or macroscopic stress relaxation [23] on a ternary Ge-As-Se mixture close to the glass-transition range which show a broad minimum about $\langle r\rangle=2.4$. The appearance of such a minimum is, however, largely governed by the strong decrease exhibited by the thermal expansivity $[12,24]$ up to the same value as well as by its further increase beyond such value.

Even more remarkable than the activation energy per se, is the finding of the dependence with connectivity of the Kohlrausch fractional exponent $\beta$ as well as its temperature coefficient. Such a parameter is seen to decrease with increasing $\langle r\rangle$ which again contrasts with results referred to above [23], which show that $\beta$ increases with average coordination up to $\langle r\rangle \approx 2.3$. Our findings come, however, into line with predictions which tell that the relaxation times $\tau$ and $\beta$ are uniquely related [19], and, in fact, analysis of some of the available data suggests that $\log (\tau)$ is a linear function of $\beta^{-1}$. The current findings show that the microscopic relaxation time and the activation energy increase with $\langle r\rangle$, a fact that is easily understandable from basic physical arguments [9]. Concomitant with such an increase here we found a decrease of $\beta$ which is understood as arising from an increase of the temperaturedependent contribution to the activation barrier.

The present results thus lend important support to some recent predictions cast within the elastic model for glasses $[5,19]$ which show that nonexponentiality is the result of some sort of cooperativity of relaxation that kicks in on lowering the temperature [18] by means of a feed-forward interaction between local relaxation events which a system uses to relax stress locally.

Work supported in part by Grant No. MAT2007-65711C-4-01, MEC, Spain. We wish to thank F. Shimojo and K.
Maruyama for providing results of their diffraction measurements on chalcogenide liquids. The authors acknowledge illuminating comments from the referees.

*Permanent address: Spallation Neutron Source, Oak Ridge, TN 37831-6465, USA.

†Present address: Forschungsneutronenquelle Heinz MaierLeibnitz (FRM-II), 85748 Garching, Germany.

[1] M. F. Thorpe, J. Non-Cryst. Solids 57, 355 (1983); J. C. Phillips, J. Non-Cryst. Solids 34, 153 (1979).

[2] M.F. Thorpe et al., in Rigidity Theory and Applications, edited by M.F. Thorpe and P. M. Duxbury (Kluwer, New York, 1999), p. 230; M. F. Thorpe et al., in Insulating and Semiconducting Glasses, edited by P. Boolchand (World Scientific, Singapore, 2000), p. 95.

[3] M. Wyart, Ann. Phys. (Paris) 30, 1 (2005).

[4] M. Micolaut and J. C. Phillips, J. Non-Cryst. Solids 353, 1732 (2007).

[5] J. C. Dyre, Rev. Mod. Phys. 78, 953 (2006).

[6] N. Xu, M. Wyart, A. J. Liu, and S. R. Nagel, Phys. Rev. Lett. 98, 175502 (2007).

[7] M. A. Briere et al., Phys. Rev. E 75, 056108 (2007); D. A. Head, J. Phys. A 37, 10771 (2004); A. Huerta and G. G. Naumis, Phys. Rev. Lett. 90, 145701 (2003).

[8] G. Foffi and F. Sciortino, Phys. Rev. E 74, 050401(R) (2006).

[9] G. G. Naumis, Phys. Rev. B 61, R9205 (2000).

[10] G. G. Naumis, J. Non-Cryst. Solids 352, 4865 (2006).

[11] T. Rouxel, Comptes Rendus Mécanique 334, 743 (2006); J. Am. Ceram. Soc. 90, 3019 (2007).

[12] M. Tatsumisago et al., Phys. Rev. Lett. 64, 1549 (1990).

[13] F. Shimojo et al., J. Non-Cryst. Solids 312-314, 349 (2002); K. Maruyama et al., J. Non-Cryst. Solids 205207, 106 (1996); M. Edeling et al., Ber. Bunsen-Ges. Phys. Chem. 85, 1049 (1981); T. S. Kavetsky et al., J. Phys. Chem. Solids 68, 712 (2007); E. Bychkov et al., Phys. Rev. B 72, 172107 (2005).

[14] J. C. Phillips, Rep. Prog. Phys. 59, 1133 (1996).

[15] R. G. Palmer et al., Phys. Rev. Lett. 53, 958 (1984); R. V. Chamberlin, Phys. Rev. Lett. 82, 2520 (1999); A. Bunde et al., Phys. Rev. Lett. 78, 3338 (1997); A. Fierro et al., Phys. Rev. E 56, 4990 (1997).

[16] E. G. D. Cohen et al., Phys. Rev. Lett. 59, 2872 (1987); M. D. Ruiz-Martin et al., Phys. Rev. B 76, 174201 (2007).

[17] F. J. Bermejo et al., Phys. Rev. B 72, 104103 (2005); M. D. Ruiz-Martin et al., Phys. Rev. B 75, 224202 (2007).

[18] K. Trachenko, Phys. Rev. B 75, 212201 (2007).

[19] K. Trachenko, C.M. Roland and R. Casalini, arXiv:0707.0416v1.

[20] L. Dagdug, L. S. Garcia-Colin, and P. Goldstein, J. NonCryst. Solids 352, 5399 (2006).

[21] W. A. Kamitakahara et al., Phys. Rev. B 44, 94 (1991).

[22] P. Boolchand et al., Solid State Ionics 39, 81 (1990).

[23] R. Böhmer and C. A. Angell, Phys. Rev. B 45, 10091 (1992).

[24] J. Ruska et al., J. Non-Cryst. Solids 22, 277 (1976); U. Senapati et al., J. Non-Cryst. Solids 185, 289 (1995). 